\title{
Exosomes and Obesity-Related Insulin Resistance
}

\author{
Li-Min Lei', Xiao Lin'2, Feng Xu', Su-Kang Shan', Bei Guo', Fu-Xing-Zi Li', \\ Ming-Hui Zheng ${ }^{1}$, Yi Wang ${ }^{1}$, Qiu-Shuang $X u^{1}$ and Ling-Qing Yuan ${ }^{1 *}$
}

\begin{abstract}
${ }^{1}$ National Clinical Research Center for Metabolic Disease, Hunan Provincial Key Laboratory of Metabolic Bone Diseases, Department of Endocrinology and Metabolism, The Second Xiangya Hospital, Central South University, Changsha, China, ${ }^{2}$ Department of Radiology, The Second Xiangya Hospital, Central South University, Changsha, China
\end{abstract}

\section{OPEN ACCESS}

Edited by:

Hamed Mirzaei,

Kashan University of Medical Sciences, Iran

Reviewed by:

Zatollah Asemi,

Kashan University of Medical

Sciences, Iran

Cecilia Bucci,

University of Salento, Italy

*Correspondence:

Ling-Qing Yuan

allenylq@csu.edu.cn

Specialty section:

This article was submitted to

Membrane Traffic,

a section of the journal

Frontiers in Cell and Developmental

Biology

Received: 11 January 2021

Accepted: 24 February 2021

Published: 18 March 2021

Citation:

Lei L-M, Lin X, Xu F, Shan S-K, Guo B, Li F-X-Z, Zheng M-H, Wang Y, Xu Q-S and Yuan L-Q (2021) Exosomes and Obesity-Related

Insulin Resistance.

Front. Cell Dev. Biol. 9:651996. doi: 10.3389/fcell.2021.651996
Exosomes are extracellular vesicles, delivering signal molecules from donor cells to recipient cells. The cargo of exosomes, including proteins, DNA and RNA, can target the recipient tissues and organs, which have an important role in disease development. Insulin resistance is a kind of pathological state, which is important in the pathogeneses of type 2 diabetes mellitus (T2DM), gestational diabetes mellitus and Alzheimer's disease. Furthermore, obesity is a kind of inducement of insulin resistance. In this review, we summarized recent research advances on exosomes and insulin resistance, especially focusing on obesity-related insulin resistance. These studies suggest that exosomes have great importance in the development of insulin resistance in obesity and have great potential for use in the diagnosis and therapy of insulin resistance.

Keywords: exosomes, insulin resistance, obesity, inflammation, mesenchymal stem cell

\section{EXOSOME}

Exosomes, which are considered extracellular vesicles (EVs), are cup-shaped structures with a diameter of 30-150 $\mathrm{nm}$ and a lipid bilayer membrane (Wortzel et al., 2019). The information molecules carried by exosomes include lipids (Carayon et al., 2011), proteins (Li et al., 2017) and nucleic acids (Kamalden et al., 2017), and these types of cargo are protected from degradation by the lipid bilayer membrane and can be transported from donor cells to recipient cells (Figure 1). There are many marker molecules to determine exosomes, including CD81, CD9, CD63, heatshock proteins (HSP60, HSP70, and HSP90), ALG-2 (apoptosis-linked gene 2)-interacting protein $\mathrm{X}$ (ALIX), and tumor susceptibility gene 101 (TSG101) (Lou et al., 2017), and glucose-regulated protein 94 (Grp94) as a negative marker (Gemel et al., 2019). As the new carrier of information exchange between cells, exosomes can be secreted by different types of cells, such as hepatocytes (Fang et al., 2018), adipocytes (Yu et al., 2018), skeletal muscle cells (Nie et al., 2019), vascular

\footnotetext{
Abbreviations: EVs, extracellular vesicles; ILVs, intraluminal vesicles; MVBs, multivesicular bodies; ALIX, ALG-2 (apoptosis-linked gene 2)-interacting protein X; TSG101, tumor susceptibility gene 101; Grp94, regulated protein 94; GDM, gestational diabetes mellitus; OGTT, oral glucose tolerance test; HOMA-IR, Homeostatic Model Assessment for Insulin Resistance; TNF- $\alpha$, tumor necrosis factor- $\alpha$; IL-6, interleukin-6; RBP4, retinol-binding protein-4; Shh, Sonic hedgehog; IRADEs, insulin resistance adipocyte-derived exosomes; IRS-1, insulin receptor substrate-1; HSL, hormone-sensitive lipase; ATM, Adipose tissue macrophage; PPAR $\gamma$, peroxisome proliferator-activated receptor $\gamma$; LPS, lipopolysaccharide; Klf4, Krüppel-like factor 4; HFD, high-fat diet; PTEN, phosphatase and tensin homolog deleted on chromosome 10; Sirt1, Sirtuin 1; FASN, fatty acid synthetase; PHLPP2, PH domain leucine-rich repeat protein phosphatase 2; GSIS, glucosestimulated insulin secretion; eWAT, epididymal white adipose tissue; A-FABP, adipocyte fatty acid-binding protein; MSCs, mesenchymal stem cells; hucMSC-ex, exosomes from human umbilical cord mesenchymal stem cells; BM-MSCs, bone marrow mesenchymal stem cells; ADSCs, adipose-derived stem cells.
} 


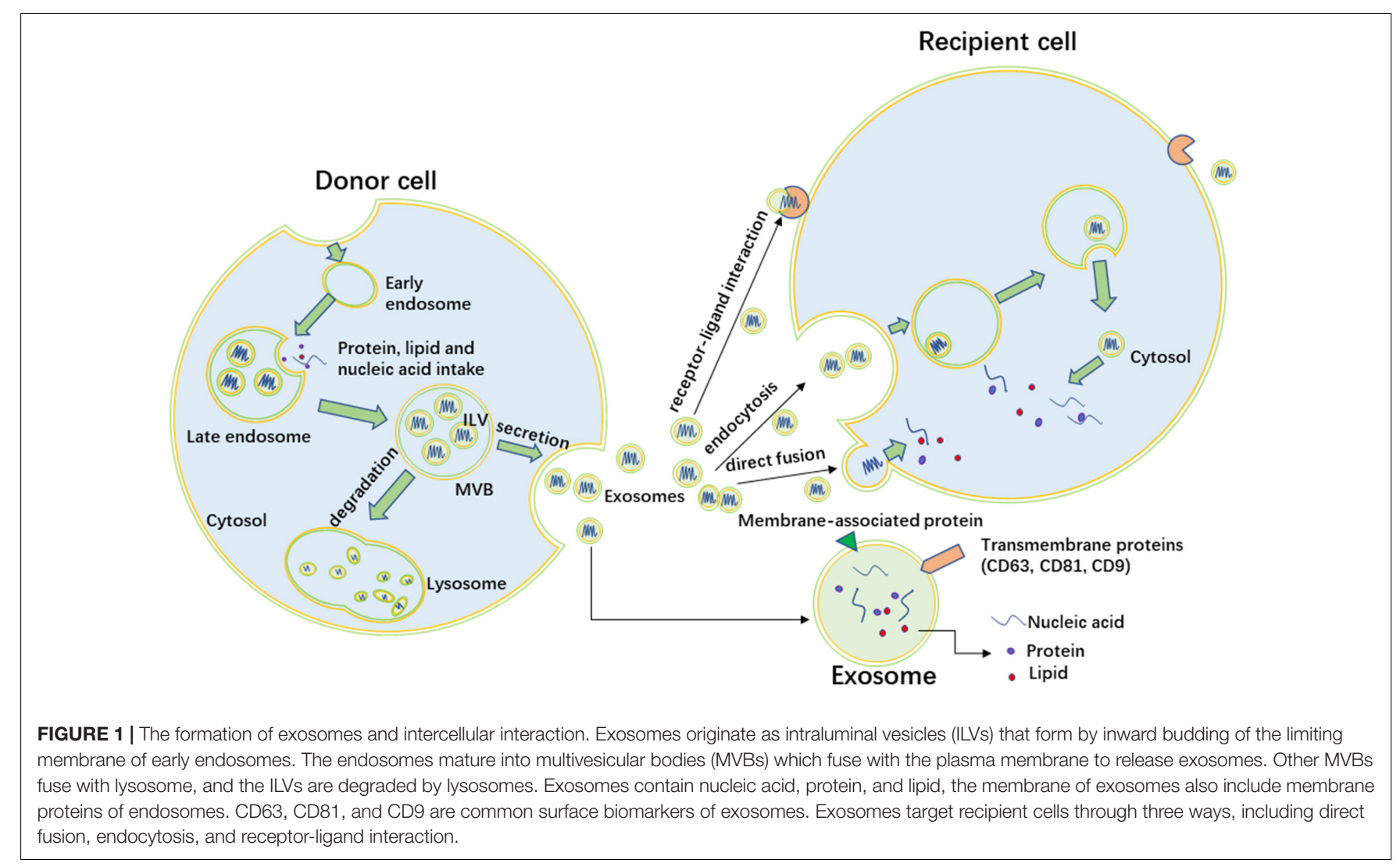

smooth muscle cells (Kapustin et al., 2015) and stem cells (Willis et al., 2018). Exosomes have been detected in various bodily fluids, including plasma, saliva, breast milk, sweat, tears, and urine (Lasser et al., 2011; McKiernan et al., 2018; Wu and Liu, 2018; Inubushi et al., 2020). What's the precise function of exosomes? Initially, exosomes were described as "garbage dumpsters," which took the useless or harmful intracellular substances out of cells. Recently, exosomes were defined as "signal boxes," which delivered messages between the cells or organs (Xie et al., 2019). Now, more and more studies have elucidated the functions of exosomes. Exosomes can participate in both pathological and physiological processes, including angiogenesis (Zeng et al., 2018), vascular calcification (Xu F. et al., 2020), immune inflammation (Pan et al., 2019), apoptosis (Guay et al., 2019), fibrosis (Seo et al., 2016), tumor development (Xue et al., 2017), senescence (Zhang et al., 2017; Xu F. et al., 2020), tissue repair (Dinh et al., 2020) and insulin resistance (Yu et al., 2018). Our present review will focus on the exosome's effect on insulin resistance.

\section{INSULIN PHYSIOLOGY AND INSULIN RESISTANCE}

As a key regulatory factor of glucose metabolism, insulin is secreted by pancreatic $\beta$ cells following elevations in blood glucose (Halperin et al., 2012). Indeed, the actions of insulin mainly contribute to glucose uptake by skeletal muscle and adipose tissue and reduce liver gluconeogenesis and glycogenolysis (Titchenell et al., 2017; Tokarz et al., 2018). Insulin also participates in protein metabolism in skeletal metabolism and lipid storage in adipose tissue (Fu et al., 2015). Recently, scientists have found that the brain was also a target tissue of insulin (Honkala et al., 2018). Insulin signaling has many complex branches, and each branch can present a kind of physiologic function. The phosphorylation of Akt takes part in most of the insulin signaling pathways, so we usually regard the level of p-AKT as a marker of insulin sensibility (Friedrichsen et al., 2010).

Insulin resistance is one of the important pathogenetic mechanisms of type 2 diabetes mellitus (T2DM) (Stenvers et al., 2018). Insulin resistance also takes a part in the development of gestational diabetes mellitus (GDM) and Alzheimer's disease (Benhalima et al., 2019; Wakabayashi et al., 2019). Insulin resistance refers to an impairment in the biological effects of insulin on target tissues, such as the ability to promote glucose uptake and inhibit the breakdown of glycogen in adipocytes and skeletal muscle cells. In addition to glucose metabolism, insulin is also involved in protein and lipid metabolism. In fact, we usually define the impairment of insulin effect on glucose metabolism as insulin resistance. When insulin resistance occurs in the body, the compensatory secretion of insulin will cause hyperinsulinaemia, resulting in a series of pathophysiological changes, such as pancreatic islet function impairment, increasing nutrient consumption and hyperlipidemia, cardiovascular damage, and finally leading to a variety of metabolic diseases, such as 
diabetes mellitus, obesity (Templeman et al., 2017) and diabetic cardiomyopathy (Jia et al., 2016). There are several methods to determine insulin resistance, such as the hyperinsulinaemic euglycaemic clamp, oral glucose tolerance test (OGTT) and Homeostatic Model Assessment for Insulin Resistance (HOMA-IR).

Among all the reasons leading to insulin resistance, obesity is one of the most significant causes of insulin resistance (Day et al., 2017). The sign of obesity is the expansion of adipose tissue, which is mainly reflected in the increase of subcutaneous adipose tissue. Subcutaneous adipose tissue is the largest warehouse of adipose tissue in humans and the preferred place for storing excess fat. However, the expanding ability of subcutaneous adipose tissue is limited. When its storage capacity is exceeded, lipids are stored in other metabolically more harmful ectopic tissues, such as the liver and skeletal muscle (Gustafson et al., 2015). Adipose tissues can store triglycerides and set free fatty acids and glycerol (Mundi et al., 2014). Free fatty acids can contribute to insulin insensitivity (Vlavcheski and Tsiani, 2018). White adipose tissue plays the most important role in storing fatty acids. By enhancing lipid synthesis or limiting lipolysis, white adipocytes store lipids to prevent toxic lipid accumulation in the liver and skeletal muscle (Czech, 2020). One study showed that the percentage of fat stored in the white adipose tissue of obese people decreased significantly, because the storage of lipids in non-adipose tissues were promoted (McQuaid et al., 2011). In contrast, brown/beige adipocytes were active in the direct uptake of glucose in response to $\beta$-adrenergic signaling and insulin, increasing energy consumption (Czech, 2020). Obesity has a detrimental effect on the function of each type of adipocyte, leading to insulin resistance (Czech, 2020). In addition to its function of storing fat, adipose tissue is also a very important endocrine organ. Adipokines, a general term for hormones secreted by adipocytes, are divided into two groups, which promote insulin actions or inhibit insulin actions, respectively (Guo et al., 2017). Leptin and adiponectin are adipokines that increase insulin sensitivity (Ayina et al., 2016). Adipose tissues can release tumor necrosis factor- $\alpha$ (TNF- $\alpha$ ) and interleukin-6 (IL-6) as inhibitory adipokines, which inhibit insulin action. In the process of adipose tissue expansion in obese people, adipose tissue produces chronic inflammation, and many M1 macrophages are recruited into adipose tissue and produce inflammatory factors. These inflammatory factors lead to insulin resistance in adipose tissues and act on other tissues to induce insulin resistance (Olefsky and Glass, 2010). All in all, adipose organ dysfunction is a key cause in the development of insulin resistance.

\section{EXOSOMES IN OBESITY-RELATED INSULIN RESISTANCE}

Obesity is the most significant risk factor for insulin resistance, but the specific pathogenesis of insulin resistance needs further study. Recently, exosomes have been proven to act as communicators between cells, which have attracted the attention of many scientists. Exosomes contain many kinds of cargo, which can influence the function of recipient cells. Researchers have revealed that exosomes participate in many kinds of disease development processes, such as liver and kidney diseases (Babuta et al., 2019; Liu et al., 2020). At the same time, the role of exosomes in insulin resistance has been studied, with many studies focusing on the effect of exosomes in obesity-related insulin resistance.

\section{The Role of Exosomes From Adipose Tissues in Obesity-Related Insulin Resistance}

Since adipose tissue plays a key role in insulin resistance, exosomes secreted from adipose tissue may be a kind of media in this process. The chronic inflammation of adipose tissue is an especially significant inducing factor in the development of insulin resistance, and the chronic inflammation manifests as macrophage infiltration. In recent years, interest has been emerging in the research of adipocyte-derived exosomes of chronic inflammation in adipose tissue (Figure 2). Deng et al. found exosomes secreted by the adipose tissue of ob/ob mice (obese model) induced the activation of macrophages through the TLR4/TRIF pathway, and the retinolbinding protein-4 (RBP4) in these exosomes played a role in the induction of macrophage activation. Furthermore, the obesity-related exosomes homed the macrophage to the liver and adipose tissues, in which macrophages secreted TNF- $\alpha$ and IL-6 to result in insulin resistance. They also confirmed the exosome-mediated macrophage impaired insulin action of myocytes. This is the first research discovering the role of exosomes in obesity-related macrophage-mediated insulin resistance (Deng et al., 2009). Song and co-workers confirmed that Sonic hedgehog (Shh) from insulin resistance adipocyte-derived exosomes (IRADEs) was the key regulator mediating M1 macrophage polarization through Ptch/PI3K signaling. They uncovered the exosomes from the IRADEtreated macrophages could contribute to insulin resistance in adipose tissues by decreasing the expression of insulin receptor substrate-1 (IRS-1) and hormone-sensitive lipase (HSL) expression. The authors gave us a new target, Shh, to inhibit the development of insulin resistance (Song et al., 2018). Adipose tissue macrophage (ATM)-derived exosomes from obese mice induced insulin resistance. However, the exosomes from lean ATMs mitigated the insulin resistance in obese mice without changing the weight of the mice. The authors revealed the key point of the effect of ATMderived exosomes in modulating insulin sensitivity was miR155 by targeting peroxisome proliferator-activated receptor $\gamma$ $(\operatorname{PPAR} \gamma)$. Furthermore, miR-155 knockout mice were much more insulin sensitive than their obese wildtype mice control counterparts (Ying et al., 2017). Similarly, Liu et al. (2019) found that exosomal miR-29a from ATMs was highly expressed in obese mice and could be transferred to adipocytes, myocytes and hepatocytes to induce insulin insistence by targeting PPAR $\delta$. A study showed exosomes from lipopolysaccharide (LPS)-activated macrophages could change the adipocyte gene expression associated with inflammation. However, the exosomes 


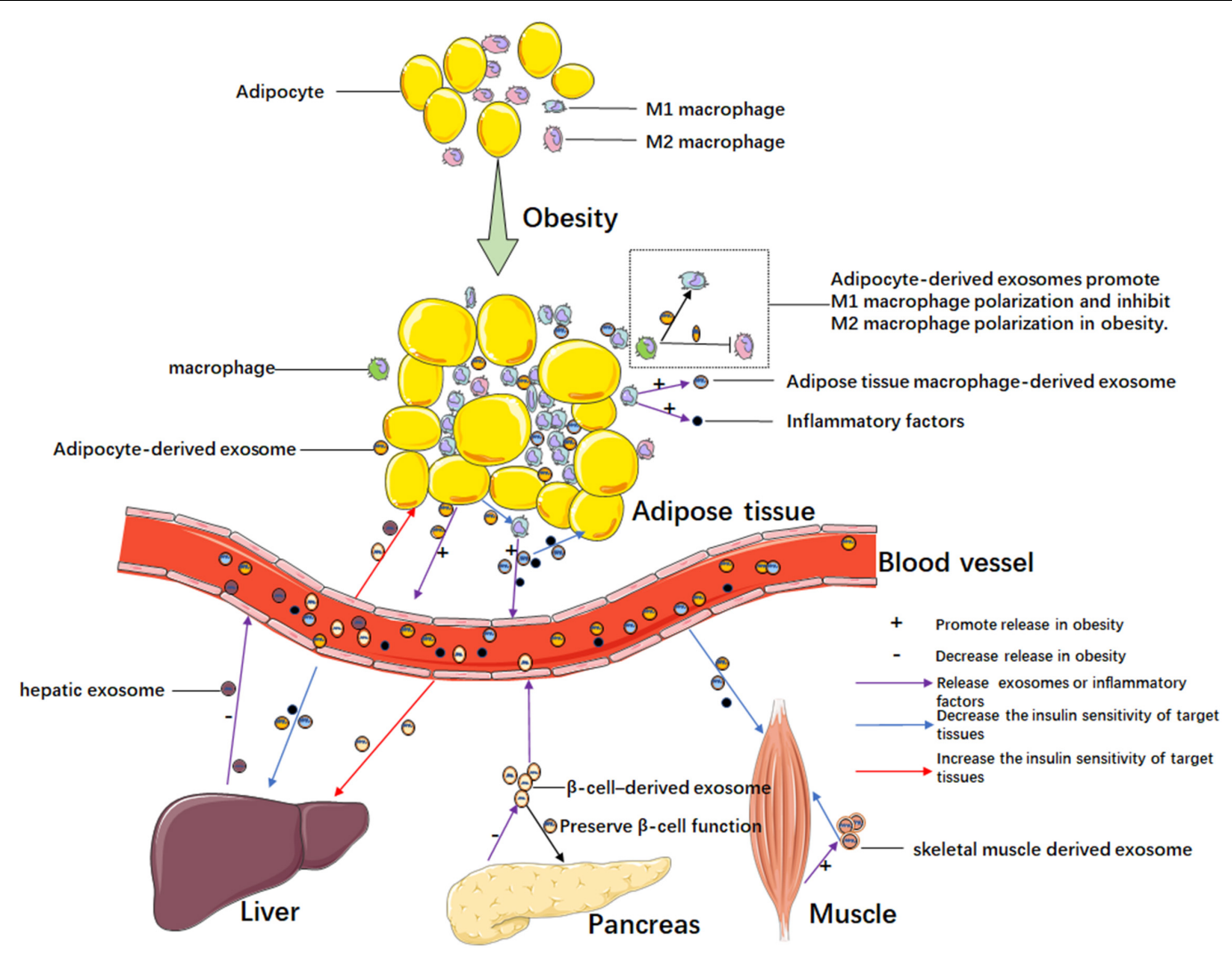

FIGURE 2 | Exosome-mediated intercellular communication in obesity-related insulin resistance. Chronic inflammation exits in adipose tissue in obesity. Adipocyte-derived exosomes promote the polarization of M1 macrophages which secretes pro-inflammatory cytokines and exosomes, and the adipose tissue macrophage-derived exosomes can promote insulin resistance in adipocytes. In obesity, exosomes from adipose tissue, liver, pancreas, and muscle, mediating intra-organ cross talks or inter-organ cross talks by blood circulation. In obesity, these organs or tissues increase the secretion of exosomes that promote insulin resistance or decrease the secretion of exosomes that ameliorate insulin resistance.

could not influence insulin-dependent glucose uptake (De Silva et al., 2018). Likewise, Pan and colleagues also confirmed that adipose tissue-related macrophage polarization was of great importance in insulin resistance. Exosomal miR-34a secreted by adipocytes inhibited M2 macrophage polarization by targeting Krüppel-like factor 4 (Klf4), which promoted M2 macrophage polarization and monocyte differentiation. The authors also verified that the altered miR-34a/Klf4 axis in visceral fat was closely associated with insulin resistance in obese subjects (Pan et al., 2019). In short, exosomes from adipocytes can increase the M1 macrophage, which secretes inflammatory cytokines, leading to insulin resistance. Apart from the inflammatory factors, ATMs also secrete exosomes to regulate insulin sensitivity. Furthermore, exosomes from macrophages can modulate adipocyte metabolism. Adipocytederived exosomes also decrease M2 macrophage levels, which is a kind of cell that inhibits inflammation.

Apart from participating in inflammation-induced insulin resistance in adipose tissues, exosomes from adipocytes also have crosstalk with other organs, inducing obesity-related insulin resistance (Figure 2). A previous study revealed crosstalk between adipose tissue and skeletal muscle tissue in obesity-related insulin resistance, and authors found that adipocyte-derived exosomal miR-27a decreased the expressions of IRS-1 and glucose transporter GLUT4 in skeletal muscle tissue by targeting PPAR $\gamma$. The authors confirmed exosomal miR-27a was derived from adipocytes, not macrophages and skeletal muscle cells (Yu et al., 2018). Dang et al. revealed that exosomes derived from adipocytes in ob/ob mice and B6 (C57BL/6j) mice fed with a high-fat diet (HFD) could decrease insulin sensitivity by affecting phosphorylation levels of AKT and glucose uptake of AML12 cells. Furthermore, the inhibition of insulin function was mainly caused by obesity in the adipose tissue of ob/ob mice, which secreted miR141-3p deficiency exosomes taken up by hepatocytes. The author confirmed that miR-141-3p could target phosphatase and tensin homolog deleted on chromosome 10 (PTEN), a negative regulator of the PI3K/AKT signaling pathway (Dang et al., 2019). In a previous study, human adipose tissue-derived EVs were revealed to inhibit hepatic insulin signaling by reducing the p-AKT levels. However, the adipose tissue-derived EVs did not have a significant effect on skeletal muscle cells compared to hepatocytes. Authors found that the level of monocyte chemoattractant protein-1 in subcutaneous adipose tissue-derived EVs and levels of IL-6 and macrophage migration inhibitory factor of omental adipose 
tissue-derived EVs were associated with AKT phosphorylation in hepatocytes (Kranendonk et al., 2014). Li et al. showed adipose-specific knockdown of Sirtuin 1 (Sirt1) contributed to obesity and insulin resistance by stimulating exosomes secretion in an autophagy-dependent manner, and adipose Sirt1 deficiency-induced exosomes affected insulin sensitivity via the TLR4/NF- $\kappa \mathrm{B}$ signaling pathway in adipose tissues ( $\mathrm{Li}$ F. et al., 2019). As adipose tissue expansion could lead to local tissue hypoxia and inflammation in obesity, Mleczko et al. revealed that the exosomes from hypoxic adipocytes impaired insulin-stimulated glucose uptake by reducing the phosphorylation of AKT. The authors also confirmed exosomes from plasma obtained from obese women could reduce insulinstimulated glucose transport. Interestingly, the inhibiting effect of the exosomes could be restored by heating the hypoxic exosomes preparation to $40^{\circ} \mathrm{C}$ for $30 \mathrm{~min}$ prior to the treatment of cells. Since the effect of hypoxic EVs on glucose transport was thermolabile, the authors guessed enzymatic activity might be responsible for this effect. Then, they found PTEN, a protein phosphatase that reduced phosphorylation of AKT existed in EVs from hypoxic 3T3L1 adipocytes. However, they did not find the precise molecular mechanisms (Mleczko et al., 2018). Hubal et al. (2017) found that gastric bypass bariatric surgery could not only reduce weight and insulin resistance but also change the microRNA content of circulating adipocyte-derived exosomes isolated from the peripheral blood. Among the altered microRNAs of exosomes, 29 microRNAs associated with insulin resistance changed after surgery; likewise, 48 microRNAs targeting 78 mRNAs were significantly correlated to branched-chain amino acid levels, which are linked to insulin dysregulation (Hubal et al., 2017). These studies suggested that exosomes from adipocytes could have a function in distant organs and take part in obesity-related insulin resistance.

\section{The Role of Exosomes Derived From Tissues Other Than Adipose Tissue in Obesity-Related Insulin Resistance}

In obesity, other organs could also produce exosomes that are associated with insulin resistance (Figure 2). A study by $\mathrm{Wu}$ et al. (2020) revealed that hepatic exosome-derived miR-130a$3 \mathrm{p}$ participated in lipid and glucose metabolism by targeting adipocytes. In HFD-induced mice, miR-130a-3p knockout mice had the highest blood lipid index and a higher blood glucose level compared to those in miR-130a-3p overexpressed and wild-type mice. Authors found hepatic exosome-derived miR130a-3p suppressed adipogenesis mainly by downregulating the expression of fatty acid synthetase (FASN) and PPAR $\gamma$ at the protein level. Besides, the authors revealed that miR130a-3p could target $\mathrm{PH}$ domain leucine-rich repeat protein phosphatase 2 (PHLPP2), increase the levels of p-AKT and p-AS160, and finally promote GLUT4 transportation (Wu et al., 2020). In a previous study, the authors confirmed the effect of skeletal muscle-derived exosomes during lipid-induced insulin resistance. In addition to insulin resistance, lipid treatment led skeletal muscle to produce more exosomes, which could increase the AKT content of recipient muscle cells, and regulate the expression of genes having to do with the cell cycle and muscle differentiation in recipient myotubes. The exosomes from lipid-treated skeletal muscle also induced myoblast proliferation. Authors also revealed that exosomes transferred lipids between muscle cells (Aswad et al., 2014). As the insulin-secreting organ, the islets are also involved in regulating insulin sensitivity. $\mathrm{Xu}$ et al. revealed that pancreatic $\beta$ cell miR-26a improved insulin sensitivity and preserved $\beta$ cell function. After confirming exosomal miR-26a was reduced in obese humans and mice, especially in the islets, the authors generated conditional transgenic mice expressing miR-26a under the control of the rat insulin promoter to increase expression of miR-26a in $\beta$ cells. The results showed that the exosomal miR-26a secreted from $\beta$ cells could increase insulin sensitivity in peripheral tissues by regulating metabolism-related gene expression, and the miR-26a could also decrease glucose-stimulated insulin secretion (GSIS) by impairing actin cytoskeleton remodeling. In addition, miR-26a ameliorated compensatory $\beta$ cell hyperplasia by decreasing $\beta$ cell replication induced by excess nutrition (Xu H. et al., 2020). In an interesting study, Wang et al. found pancreatic cancer-derived exosomes also inhibited glucose intake in $\mathrm{C} 2 \mathrm{C} 12$ myotube cells through the PI3K/AKT/FoxO1 pathway. The increase of FoxO1 caused by pancreatic cancerderived exosomes contributed to inhibiting the translocation of GLUT4 to the plasma membrane. Although the authors showed exosomal miRNA might be involved in this process through microRNA microarray analysis, they did not confirm the concrete molecular mechanism (Wang et al., 2017). A study by Choi et al. (2015) confirmed that EVs from gut microbes induced by a high fat diet (HFD) could impair insulin signaling and glucose metabolism both in vitro and in vivo. They observed that the HFD changed the bacteria in the gut. However, the gut bacteria did not infiltrate through the gut to other organs, but the gut microbe-derived EVs caused insulin resistance by infiltrating the gut barrier and targeting other organs (Choi et al., 2015). In a previous study, researchers determined the circulating exosome miRNA profile in dietinduced central obesity mice and found an increase in miR122, miR-192, miR-27a-3p, and miR-27b-3p. As the exosomes from obese mice could induce insulin resistance, authors used exosomes transfected with obesity-associated miRNA mimic injected into lean mice to exclude other sources of variation. The results showed that mimic treatment targeted PPAR $\gamma$ to induce inflammation and hepatic steatosis in epididymal white adipose tissue (eWAT), both of which are known to participate in glucose intolerance and dyslipidemia, and the effects of mimic treatment could be reverted by the lipolysis inhibitor acipimox or the PPAR $\alpha$ agonist fenofibrate (Castaño et al., 2018). Likewise, a clinical study showed that the number of circulating EVs was strongly associated with obesity and lipid and glucose metabolism. Furthermore, some EVs were confirmed from adipocytes and hepatocytes, which are major metabolic cells (Kobayashi et al., 2018).

Above all, insulin resistance is a complicated pathological state, which affects various organs. Furthermore, exosomes play a pivotal role in the development of obesity-related insulin 
resistance. Above all, the function of exosomal contents can be seen in Table 1.

\section{EXOSOMES AS BIOMARKERS FOR INSULIN RESISTANCE}

The OGTT and HOMA-IR are useful to test for insulin resistance, although the hyperinsulinaemic euglycaemic clamp is regarded as the gold standard for the diagnosis of insulin resistance diagnosis. However, it can't be used as a routine test. Recently, many biomarkers for insulin resistance have been revealed. Adipokines could be regarded as biomarkers for insulin resistance, including adiponectin, RBP4, chemerin, and adipocyte fatty acid-binding protein (A-FABP) (Huang et al., 2013; Li et al., 2018;
Li X. et al., 2019; Frithioff-Bojsoe et al., 2020). Fibroblast growth factor 21 and fetuin-A have been found as biomarkers for insulin resistance (Shim et al., 2017; Xu et al., 2018). Many studies have shown that myokines, such as IL-6, irisin and myostatin, serve as biomarkers for insulin resistance (Mauer et al., 2014; Steculorum et al., 2016; Mazur-Bialy, 2019).

The release of exosomes depends on the state of the human body. What's more, exosomes can protect the cargo from degradation by the lipid bilayer membrane. Therefore, collecting exosomes is a potential promising diagnostic method for diseases. Exosomes were found in various bodily fluids, such as plasma, saliva, breast milk, sweat, tears, and urine. Therefore, exosomes can easily be obtained from bodily fluids. It has been reported that exosomes are of great significance for fluid biopsy in many kinds of diseases, such as breast cancer (Hannafon et al., 2016),

TABLE 1 | Role of exosomes in obesity-related insulin resistance.

\begin{tabular}{|c|c|c|c|c|}
\hline Source & Contents & Functions & Level & References \\
\hline Adipocytes & RBP4 & $\begin{array}{l}\text { Activation of macrophage impairing glucose uptake and the } \\
\text { insulin response depending on the TLR } 4 \text { pathway and } \\
\text { inducing TNF- } \alpha \text { and IL-6 }\end{array}$ & Overexpress & Deng et al., 2009 \\
\hline Insulin resistance adipocytes & $\begin{array}{l}\text { Sonic Hedgehog } \\
\text { (Shh) }\end{array}$ & $\begin{array}{l}\text { Mediating M1 macrophage polarization through Ptch/PI3K } \\
\text { signaling and educating macrophage which produces } \\
\text { exosomes causing insulin resistance by decreasing the } \\
\text { expression of IRS-1 and HSL expression }\end{array}$ & Overexpress & Song et al., 2018 \\
\hline Adipocytes & microRNA-34a & Inhibiting M2 macrophage polarization by targeting Klf4 & Overexpress & Pan et al., 2019 \\
\hline Adipose tissue macrophages & miR-155 & Impairing insulin sensitivity by targeting PPAR $\gamma$ & Overexpress & Ying et al., 2017 \\
\hline Adipose tissue macrophages & miR-29a & $\begin{array}{l}\text { Transferring to adipocytes, myocytes, and hepatocytes to } \\
\text { induce insulin insistence by targeting PPAR- } \delta\end{array}$ & Overexpress & Liu et al., 2019 \\
\hline Adipocytes & $\operatorname{miR}-27 a$ & $\begin{array}{l}\text { Decreasing the expressions of IRS- } 1 \text { and GLUT4 in skeletal } \\
\text { muscle tissue by targeting PPAR } \gamma\end{array}$ & Overexpress & Yu et al., 2018 \\
\hline Adipocytes & $\operatorname{miR}-141-3 p$ & $\begin{array}{l}\text { Increasing PI3K/AKT signaling pathway by targeting PTEN } \\
\text { in hepatocytes }\end{array}$ & Downexpress & Dang et al., 2019 \\
\hline Adipocytes & Sirt1 & $\begin{array}{l}\text { Decreasing insulin resistance by reducing TLR4/NF-кB } \\
\text { signaling pathway }\end{array}$ & Downexpress & Li F. et al., 2019 \\
\hline Hypoxic adipocytes & - & $\begin{array}{l}\text { Impairing insulin-stimulated glucose uptake by reducing } \\
\text { AKT phosphorylation }\end{array}$ & - & Mleczko et al., 2018 \\
\hline Hepatocytes & miR-130a-3p & $\begin{array}{l}\text { Suppressing adipogenesis by downregulating the } \\
\text { expression of FASN and PPAR } \gamma \text { at the protein level and } \\
\text { increasing the level of P-AKT and P-AS160 by targeting } \\
\text { PHLPP2 }\end{array}$ & Downexpress & Wu et al., 2020 \\
\hline Pancreatic $\beta$ cells & microRNA-26a & $\begin{array}{l}\text { Increasing insulin sensitivity in peripheral tissues, decreasing } \\
\text { glucose-stimulated insulin secretion (GSIS) by impairing } \\
\text { actin cytoskeleton remodeling and preserving } \beta \text { cell function }\end{array}$ & Downexpress & Xu H. et al., 2020 \\
\hline Gut microbes & - & $\begin{array}{l}\text { Inducing insulin resistance by infiltrating the gut barrier and } \\
\text { targeting other organs }\end{array}$ & - & Choi et al., 2015 \\
\hline Serum & $\begin{array}{l}\operatorname{miR}-122, \text { miR-192, } \\
\text { miR-27a-3p, and } \\
\text { miR-27b-3p }\end{array}$ & $\begin{array}{l}\text { Targeting Pparg to induce eWAT Inflammation and hepatic } \\
\text { steatosis }\end{array}$ & Overexpress & Castaño et al., 2018 \\
\hline
\end{tabular}

TABLE 2 | Potential biomarkers of exosomes in insulin resistance.

\begin{tabular}{|c|c|c|c|}
\hline Source & Potential biomarker & Express level & References \\
\hline Urine exosomes & Phosphoenolpyruvate carboxykinase & Up & Sharma et al., 2020 \\
\hline Plasma exosomes & Let-7b, miR-144-5p, miR-34a, and miR-532-5p & Up & Jones et al., 2017 \\
\hline Serum exosomes & miR-20b-5p & Up & Katayama et al., 2019 \\
\hline Neural origin plasma exosomes & pSer312-IRS-1 and p-panTyr-IRS-1 & higher pSer312-IRS-1 and lower p-panTyr-IRS-1 & Mullins et al., 2017 \\
\hline
\end{tabular}




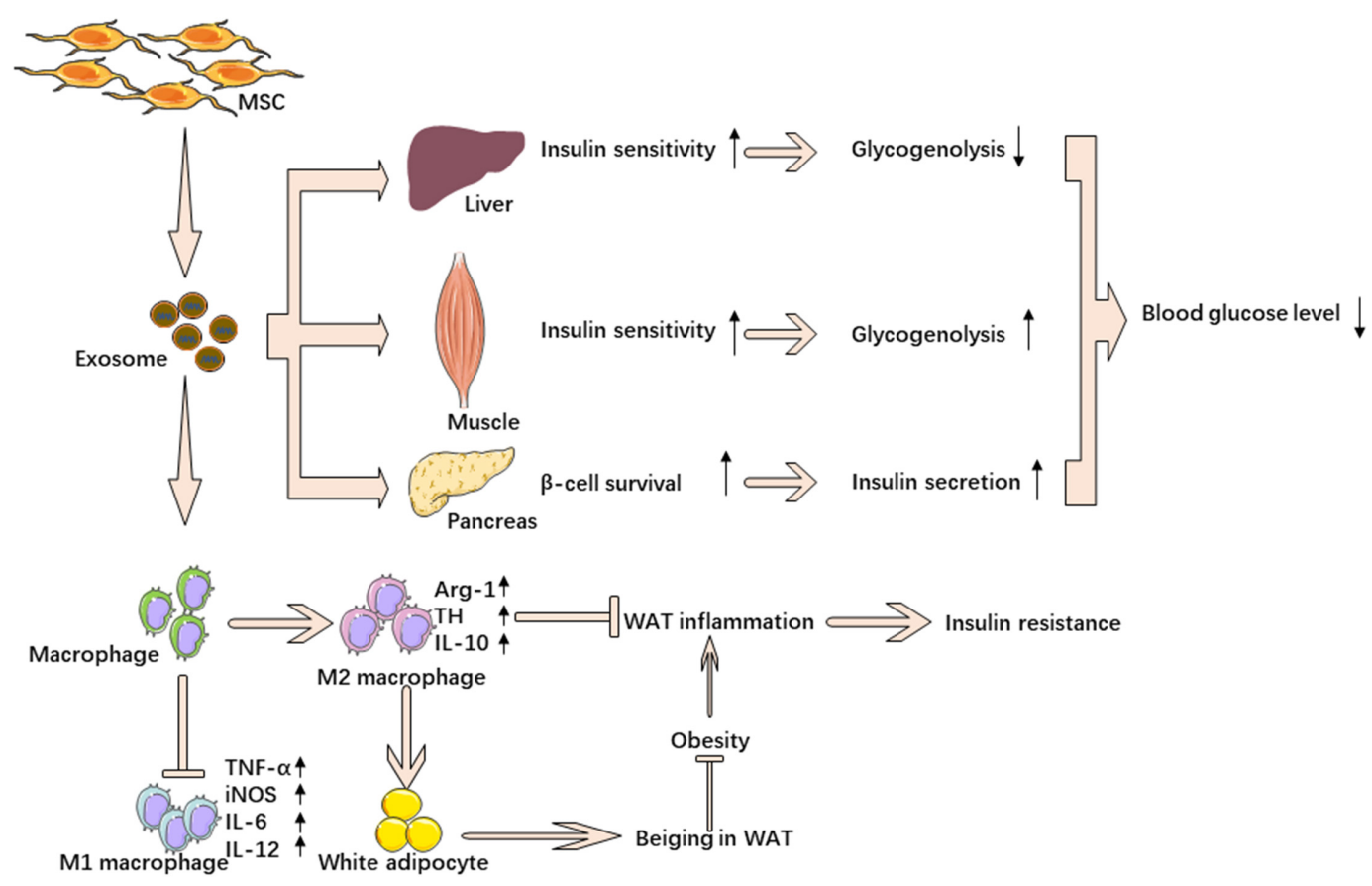

FIGURE 3 | MSC-derived exosomes ameliorate insulin resistance. MSC-derived exosomes down regulate blood glucose through reverse insulin resistance in insulin target tissue and relieve $\beta$ cell destruction. MSC-derived exosomes also ameliorate insulin resistance by promoting M2 macrophage polarization and inhibiting M1 macrophage polarization.

hepatocellular carcinoma (Sohn et al., 2015), prostate cancer (Bhagirath et al., 2018), osteoarthritis (Zhao and Xu, 2018) and atherosclerosis (Lu et al., 2019). Likewise, using exosomes for the early diagnosis of insulin resistance is a promising method. Sharma et al. reported that the phosphoenolpyruvate carboxykinase in urine exosomes reflected gluconeogenesis of the kidney. By determining phosphoenolpyruvate carboxykinase, urine exosomes, as a non-invasive marker, contribute to discovering impairment in gluconeogenesis and early insulin resistance in humans (Sharma et al., 2020). A clinical study showed that circulating miRNAs in the EVs of human plasma could be biomarkers for insulin resistance phenotypes in obesity. Authors found that four miRNAs (let-7b, miR-144-5p, miR-34a, and miR-532-5p) were strongly predictive of insulin resistance (Jones et al., 2017). Another study by Katayama et al. (2019) revealed that miRNA expression profiles in exosomes, rather than in serum, had a significant difference between normal glucose tolerance and patients with T2DM. The increase in circulating exosomal miR-20b-5p was confirmed to impair insulin signaling in human skeletal muscle by targeting AKTIP and STAT3 (Katayama et al., 2019). Brain insulin resistance exists in Alzheimer's disease. A study showed neurally derived blood exosomes were used to track the dysfunctional phosphorylation type 1 insulin receptor, which could happen in preclinical Alzheimer's disease (Kapogiannis et al., 2015). Mullins et al. (2017) found that neural origin plasma exosomes labeled by L1CAM contained higher pSer312-IRS-1 (ineffective insulin signaling) and lower p-panTyr-IRS-1 (effective insulin signaling) in Alzheimer's disease. Furthermore, they revealed that higher
pSer312-IRS-1 levels were positively related to greater brain atrophy in Alzheimer's disease and p-panTyr-IRS-1 levels had the opposite effect (Mullins et al., 2017). In a word, exosomes will be the most promising bodily fluid biopsy. As shown in Table 2, some potential exosomal biomarkers can be used to track insulin resistance.

\section{MESENCHYMAL STEM CELLS-DERIVED EXOSOMES IN INSULIN RESISTANCE}

Mesenchymal stem cells (MSCs) are multipotent cells that can be self-renewing, including human umbilical cord mesenchymal stem cells, bone marrow mesenchymal stem cells and adiposederived stem cells. Recently, stem cell therapy has been a promising strategy for diseases, such as liver disease (Zhao L. et al., 2018), multiple sclerosis (Muraro et al., 2017; Atkins, 2019), leukemia (Shem-Tov et al., 2020) and diabetic retinopathy (Gaddam et al., 2019). However, whether stem cell transplantation contributes to the emergence of tumors remains unknown. It has been found that stem cells have an effect on tissue-resident recipient cells by paracrine mechanisms (Liang et al., 2014). It has been reported that exosomes secreted by mesenchymal stem cells have the properties of their parent cells, such as regulation of cell migration and proliferation (McBride et al., 2017; Chew et al., 2019), immunomodulation (Willis et al., 2018), tissue regeneration (Chew et al., 2019) and antiinflammatory (Xia et al., 2019) effects. 
Several researchers investigated the effect of mesenchymal stem cell-derived exosomes in insulin resistance. Sun et al. showed that exosomes from human umbilical cord mesenchymal stem cells (hucMSC-ex) could have a therapeutic effect on T2DM. They found that injection of hucMSC-ex significantly ameliorated hyperglycemia in rats with T2DM. HucMSC-ex could increase insulin sensitivity by increasing the activation of p-IRS-1 and p-AKT and inhibiting the secretion of proinflammatory cytokines, which could inhibit the activation of the insulin signaling pathway. These exosomes could promote glucose uptake and glycolysis in skeletal muscle by affecting membrane translocation of GLUT4 and glucose metabolismrelated enzymes. Activating insulin signaling, hucMSC-ex increased expression of $\mathrm{p}-\mathrm{GSK} 3 \beta$ and glycogen synthase to improve glycogen synthesis in the liver. Furthermore, hucMSCex could not only promote the secretion of insulin but also inhibit STZ-induced $\beta$ cell apoptosis (Sun et al., 2018). However, this research didn't explain which kind of substance contained in hucMSC-ex played a role in the process. In a previous study, Su et al. (2019) found exosomes from bone marrow mesenchymal stem cells (BM-MSCs) of aged mice impaired insulin sensitivity both in vitro and in vivo. After a miRNA microarray analysis, the authors paid attention to miR-29b-3p, which was significantly higher in exosomes secreted by the BMMSCs of aged mice compared to that released by the BM-MSCs of young mice. They revealed that Sirt1 was downstream of miR-29b-3p and regulated insulin sensitivity. Injection of the BM-MSC-specific nanocomplex/aptamer-agomiR-29b-3p to the bone marrow cavity could impair insulin sensitivity in young mice. On the contrary, BM-MSC-specific nanocomplex/aptamerantagomiR-29b-3p mitigated aging-associated resistance in old mice. However, they did not find strong evidence showing BM-MSC-derived exosomes affect aging-associated degeneration of pancreatic function ( $\mathrm{Su}$ et al., 2019). Zhao et al. found that exosomes from adipose-derived stem cells (ADSCs) were taken up by macrophages. Furthermore, these exosomes carried active STAT3, which could promote arginase-1 expression in macrophages to induce anti-inflammatory M2 phenotypes. ADSC-derived exosomes mitigated diet-induced obesity and improved glucose tolerance and insulin sensitivity. Besides, the authors found that ADSC-derived exosomes could inhibit adipocyte hypertrophy and promote the occurrence of brownlike fats. More interestingly, the results showed that macrophages treated by ADSC-derived exosomes could induce ADSC proliferation by secreting lactate (Zhao H. et al., 2018). Taken together, stem cell-derived exosomes could increase insulin sensitivity by promoting insulin signaling and decreasing adipose tissue-related inflammation (Figure 3).

\section{SUMMARY AND PERSPECTIVES}

Obesity is of great importance in the development of insulin resistance. The chronic inflammation in adipose tissues is the main cause of insulin resistance in obesity, and great importance in the development of inflammation has been attached to macrophages. Exosomes, as the mediators, delivering the contents from parent cells to recipient cells, affect the pathophysiology of human beings. In this review, we summarized a lot of exosome-associated research, which mainly focuses on obesity-related insulin resistance. Adipocyte-derived exosomes participate in the activation of macrophages by promoting M1 macrophage polarization and inhibiting M2 macrophage polarization and subsequently stimulating insulin resistance. Exosomes from activated macrophages also induce insulin resistance. Furthermore, exosomes derived from tissues other than adipose tissue also play a role in obesity-related insulin resistance.

Exosomes could be potential diagnostic and therapeutic tools for insulin resistance. Liquid biopsy is a very valuable test, and it reflects the overall physical condition. Bodily fluids contain a variety of substances, so it is important to choose suitable and specific biomarkers. The exosome is a potential biomarker for insulin resistance, since it can be changed by the body's pathophysiological state. As the exosome is a membrane structure that can protect the internal molecules from degradation, we can use exosomes to detect changes in the nucleic acid levels of patients. Although the application of exosomes as a detection method is not very common at present, with in-depth research on exosomes, people will better understand the superiority of exosomes as a tool for liquid biopsy. The application of exosomes from mesenchymal stem cells has been studied for many years. The effect of MSC-exosome treatment on insulin resistance has been confirmed in several studies. Exosomes have great advantages for the treatment of insulin resistance. Firstly, exosomes have the physiological activity of their parent cells and can replace the parent cells to play a therapeutic role. Secondly, exosomes are less immunogenic and do not cause immunologic rejection in patients. Thirdly, exosomes are vesicle structures that do not pose a tumorigenic risk to organisms. Fourthly, exosomes can treat insulin resistance by loading some drugs. Although the current research on the diagnosis and treatment of insulin resistance with exosomes is only the tip of the iceberg. In the future, using advanced technology and methods, we will skilfully use exosomes to diagnose and treat insulin resistance.

\section{AUTHOR CONTRIBUTIONS}

L-QY: manuscript writing and approving the final version of manuscript. L-ML: study conduct, data analysis, and manuscript writing. XL, FX, S-KS, BG, F-X-ZL, M-HZ, YW, and Q-SX: data analysis. All authors: reviewed the manuscript.

\section{FUNDING}

This work was supported by funding from the National Natural Science Foundation of China (Nos. 81770881 and 82070910) and Key R\&D Plan of Hunan Province (2020SK2078). 


\section{REFERENCES}

Aswad, H., Forterre, A., Wiklander, O. P., Vial, G., Danty-Berger, E., Jalabert, A., et al. (2014). Exosomes participate in the alteration of muscle homeostasis during lipid-induced insulin resistance in mice. Diabetologia 57, 2155-2164. doi: 10.1007/s00125-014-3337-2

Atkins, H. (2019). Stem cell transplantation to treat multiple sclerosis. Jama 321, 153-155. doi: 10.1001/jama.2018.20777

Ayina, C. N., Noubiap, J. J., Etoundi Ngoa, L. S., Boudou, P., Gautier, J. F., Mengnjo, M. K., et al. (2016). Association of serum leptin and adiponectin with anthropomorphic indices of obesity, blood lipids and insulin resistance in a Sub-Saharan African population. Lipids Health Dis 15:96. doi: 10.1186/s12944016-0264-x

Babuta, M., Furi, I., Bala, S., Bukong, T. N., Lowe, P., Catalano, D., et al. (2019). Dysregulated autophagy and lysosome function are linked to exosome production by Micro-RNA 155 in alcoholic liver disease. Hepatology 70, 2123 2141. doi: 10.1002/hep.30766

Benhalima, K., Van Crombrugge, P., Moyson, C., Verhaeghe, J., Vandeginste, S., Verlaenen, H., et al. (2019). Characteristics and pregnancy outcomes across gestational diabetes mellitus subtypes based on insulin resistance. Diabetologia 62, 2118-2128. doi: 10.1007/s00125-019-4961-7

Bhagirath, D., Yang, T. L., Bucay, N., Sekhon, K., Majid, S., Shahryari, V., et al. (2018). microRNA-1246 Is an exosomal biomarker for aggressive prostate cancer. Cancer Res. 78, 1833-1844. doi: 10.1158/0008-5472.Can-17-2069

Carayon, K., Chaoui, K., Ronzier, E., Lazar, I., Bertrand-Michel, J., Roques, V., et al. (2011). Proteolipidic composition of exosomes changes during reticulocyte maturation. J. Biol. Chem. 286, 34426-34439. doi: 10.1074/jbc.M111.257444

Castaño, C., Kalko, S., Novials, A., and Párrizas, M. (2018). Obesity-associated exosomal miRNAs modulate glucose and lipid metabolism in mice. Proc. Natl. Acad. Sci. U.S.A. 115, 12158-12163. doi: 10.1073/pnas.1808855115

Chew, J. R. J., Chuah, S. J., Teo, K. Y. W., Zhang, S., Lai, R. C., Fu, J. H., et al. (2019). Mesenchymal stem cell exosomes enhance periodontal ligament cell functions and promote periodontal regeneration. Acta Biomater. 89, 252-264. doi: 10.1016/j.actbio.2019.03.021

Choi, Y., Kwon, Y., Kim, D. K., Jeon, J., Jang, S. C., Wang, T., et al. (2015). Gut microbe-derived extracellular vesicles induce insulin resistance, thereby impairing glucose metabolism in skeletal muscle. Sci. Rep. 5:15878. doi: 10. $1038 /$ srep 15878

Czech, M. P. (2020). Mechanisms of insulin resistance related to white, beige, and brown adipocytes. Mol. Metab. 34, 27-42. doi: 10.1016/j.molmet.2019.12.014

Dang, S. Y., Leng, Y., Wang, Z. X., Xiao, X., Zhang, X., Wen, T., et al. (2019). Exosomal transfer of obesity adipose tissue for decreased miR-141-3p mediate insulin resistance of hepatocytes. Int. J. Biol. Sci. 15, 351-368. doi: 10.7150/ijbs. 28522

Day, S. E., Coletta, R. L., Kim, J. Y., Garcia, L. A., Campbell, L. E., Benjamin, T. R., et al. (2017). Potential epigenetic biomarkers of obesity-related insulin resistance in human whole-blood. Epigenetics 12, 254-263. doi: 10.1080/ 15592294.2017.1281501

De Silva, N., Samblas, M., Martinez, J. A., and Milagro, F. I. (2018). Effects of exosomes from LPS-activated macrophages on adipocyte gene expression, differentiation, and insulin-dependent glucose uptake. J. Physiol. Biochem. 74, 559-568. doi: 10.1007/s13105-018-0622-4

Deng, Z. B., Poliakov, A., Hardy, R. W., Clements, R., Liu, C., Liu, Y., et al. (2009). Adipose tissue exosome-like vesicles mediate activation of macrophageinduced insulin resistance. Diabetes 58, 2498-2505. doi: 10.2337/db09-0216

Dinh, P. C., Paudel, D., Brochu, H., Popowski, K. D., Gracieux, M. C., Cores, J., et al. (2020). Inhalation of lung spheroid cell secretome and exosomes promotes lung repair in pulmonary fibrosis. Nat. Commun. 11:1064. doi: 10.1038/s41467-02014344-7

Fang, J. H., Zhang, Z. J., Shang, L. R., Luo, Y. W., Lin, Y. F., Yuan, Y., et al. (2018). Hepatoma cell-secreted exosomal microRNA-103 increases vascular permeability and promotes metastasis by targeting junction proteins. Hepatology 68, 1459-1475. doi: 10.1002/hep.29920

Friedrichsen, M., Poulsen, P., Richter, E. A., Hansen, B. F., Birk, J. B., RibelMadsen, R., et al. (2010). Differential aetiology and impact of phosphoinositide 3-kinase (PI3K) and Akt signalling in skeletal muscle on in vivo insulin action. Diabetologia 53, 1998-2007. doi: 10.1007/s00125-010-1795-8
Frithioff-Bojsoe, C., Lund, M. A. V., Lausten-Thomsen, U., Hedley, P. L., Pedersen, O., Christiansen, M., et al. (2020). Leptin, adiponectin, and their ratio as markers of insulin resistance and cardiometabolic risk in childhood obesity. Pediatr. Diabetes 21, 194-202. doi: 10.1111/pedi.12964

Fu, X., Dong, B., Tian, Y., Lefebvre, P., Meng, Z., Wang, X., et al. (2015). MicroRNA-26a regulates insulin sensitivity and metabolism of glucose and lipids. J. Clin. Invest. 125, 2497-2509. doi: 10.1172/JCI75438

Gaddam, S., Periasamy, R., and Gangaraju, R. (2019). Adult stem cell therapeutics in diabetic retinopathy. Int. J. Mol. Sci. 20:4876. doi: 10.3390/ijms20194876

Gemel, J., Kilkus, J., Dawson, G., and Beyer, E. C. (2019). Connecting exosomes and connexins. Cancers 11:476. doi: 10.3390/cancers 11040476

Guay, C., Kruit, J. K., Rome, S., Menoud, V., Mulder, N. L., Jurdzinski, A., et al. (2019). Lymphocyte-derived exosomal MicroRNAs promote pancreatic beta cell death and may contribute to type 1 diabetes development. Cell Metab. 29, 348.e6-361.e6. doi: 10.1016/j.cmet.2018.09.011

Guo, M., Li, C., Lei, Y., Xu, S., Zhao, D., and Lu, X. Y. (2017). Role of the adipose PPARgamma-adiponectin axis in susceptibility to stress and depression/anxiety-related behaviors. Mol. Psychiatry 22, 1056-1068. doi: 10. $1038 / \mathrm{mp} .2016 .225$

Gustafson, B., Hedjazifar, S., Gogg, S., Hammarstedt, A., and Smith, U. (2015). Insulin resistance and impaired adipogenesis. Trends Endocrinol. Metab. 26, 193-200. doi: 10.1016/j.tem.2015.01.006

Halperin, F., Lopez, X., Manning, R., Kahn, C. R., Kulkarni, R. N., and Goldfine, A. B. (2012). Insulin augmentation of glucose-stimulated insulin secretion is impaired in insulin-resistant humans. Diabetes 61, 301-309. doi: 10.2337/db111067

Hannafon, B. N., Trigoso, Y. D., Calloway, C. L., Zhao, Y. D., Lum, D. H., Welm, A. L., et al. (2016). Plasma exosome microRNAs are indicative of breast cancer. Breast. Cancer Res. 18:90. doi: 10.1186/s13058-016-0753-x

Honkala, S. M., Johansson, J., Motiani, K. K., Eskelinen, J. J., Virtanen, K. A., Loyttyniemi, E., et al. (2018). Short-term interval training alters brain glucose metabolism in subjects with insulin resistance. J. Cereb. Blood Flow Metab. 38, 1828-1838. doi: 10.1177/0271678X17734998

Huang, C. L., Wu, Y. W., Hsieh, A. R., Hung, Y. H., Chen, W. J., and Yang, W. S. (2013). Serum adipocyte fatty acid-binding protein levels in patients with critical illness are associated with insulin resistance and predict mortality. Crit. Care 17:R22. doi: 10.1186/cc12498

Hubal, M. J., Nadler, E. P., Ferrante, S. C., Barberio, M. D., Suh, J. H., Wang, J., et al. (2017). Circulating adipocyte-derived exosomal MicroRNAs associated with decreased insulin resistance after gastric bypass. Obesity 25, 102-110. doi: 10.1002/oby.21709

Inubushi, S., Kawaguchi, H., Mizumoto, S., Kunihisa, T., Baba, M., Kitayama, Y., et al. (2020). Oncogenic miRNAs identified in tear exosomes from metastatic breast cancer patients. Anticancer Res. 40, 3091-3096. doi: 10.21873/anticanres. 14290

Jia, G., DeMarco, V. G., and Sowers, J. R. (2016). Insulin resistance and hyperinsulinaemia in diabetic cardiomyopathy. Nat. Rev. Endocrinol. 12, 144153. doi: $10.1038 /$ nrendo.2015.216

Jones, A., Danielson, K. M., Benton, M. C., Ziegler, O., Shah, R., Stubbs, R. S., et al. (2017). miRNA signatures of insulin resistance in obesity. Obesity 25, 1734-1744. doi: 10.1002/oby.21950

Kamalden, T. A., Macgregor-Das, A. M., Kannan, S. M., Dunkerly-Eyring, B., Khaliddin, N., Xu, Z., et al. (2017). Exosomal MicroRNA-15a transfer from the pancreas augments diabetic complications by inducing oxidative stress. Antioxid Redox Signal. 27, 913-930. doi: 10.1089/ars.2016.6844

Kapogiannis, D., Boxer, A., Schwartz, J. B., Abner, E. L., Biragyn, A., Masharani, U., et al. (2015). Dysfunctionally phosphorylated type 1 insulin receptor substrate in neural-derived blood exosomes of preclinical Alzheimer's disease. FASEB J. 29, 589-596. doi: 10.1096/fj.14-262048

Kapustin, A. N., Chatrou, M. L., Drozdov, I., Zheng, Y., Davidson, S. M., Soong, D., et al. (2015). Vascular smooth muscle cell calcification is mediated by regulated exosome secretion. Circ. Res. 116, 1312-1323. doi: 10.1161/CIRCRESAHA.116. 305012

Katayama, M., Wiklander, O. P. B., Fritz, T., Caidahl, K., El-Andaloussi, S., Zierath, J. R., et al. (2019). Circulating exosomal miR-20b-5p Is Elevated in Type 2 diabetes and could impair insulin action in human skeletal muscle. Diabetes 68, 515-526. doi: $10.2337 / \mathrm{db} 18-0470$ 
Kobayashi, Y., Eguchi, A., Tempaku, M., Honda, T., Togashi, K., Iwasa, M., et al. (2018). Circulating extracellular vesicles are associated with lipid and insulin metabolism. Am. J. Physiol. Endocrinol. Metab. 315, E574-E582. doi: 10.1152/ ajpendo.00160.2018

Kranendonk, M. E., Visseren, F. L., van Herwaarden, J. A., Nolte-'t Hoen, E. N., de Jager, W., Wauben, M. H., et al. (2014). Effect of extracellular vesicles of human adipose tissue on insulin signaling in liver and muscle cells. Obesity 22, 2216-2223. doi: 10.1002/oby.20847

Lasser, C., Alikhani, V. S., Ekstrom, K., Eldh, M., Paredes, P. T., Bossios, A., et al. (2011). Human saliva, plasma and breast milk exosomes contain RNA: uptake by macrophages. J. Transl. Med. 9:9. doi: 10.1186/1479-5876-9-9

Li, F., Li, H., Jin, X., Zhang, Y., Kang, X., Zhang, Z., et al. (2019). Adiposespecific knockdown of Sirt1 results in obesity and insulin resistance by promoting exosomes release. Cell Cycle 18, 2067-2082. doi: 10.1080/15384101. 2019.1638694

Li, X., Zhu, Q., Wang, W., Qi, J., He, Y., Wang, Y., et al. (2019). Elevated chemerin induces insulin resistance in human granulosa-lutein cells from polycystic ovary syndrome patients. Faseb J. 33, 11303-11313. doi: 10.1096/fj.201802829R

Li, G., Esangbedo, I. C., Xu, L., Fu, J., Li, L., Feng, D., et al. (2018). Childhood retinol-binding protein 4 (RBP4) levels predicting the 10-year risk of insulin resistance and metabolic syndrome: the BCAMS study. Cardiovasc. Diabetol. 17:69. doi: 10.1186/s12933-018-0707-y

Li, W., Li, C., Zhou, T., Liu, X., Liu, X., Li, X., et al. (2017). Role of exosomal proteins in cancer diagnosis. Mol. Cancer 16:145. doi: 10.1186/s12943-017-0706-8

Liang, X., Ding, Y., Zhang, Y., Tse, H. F., and Lian, Q. (2014). Paracrine mechanisms of mesenchymal stem cell-based therapy: current status and perspectives. Cell Transplant 23, 1045-1059. doi: 10.3727/096368913x667709

Liu, T., Sun, Y. C., Cheng, P., and Shao, H. G. (2019). Adipose tissue macrophagederived exosomal miR-29a regulates obesity-associated insulin resistance. Biochem. Biophys. Res. Commun. 515, 352-358. doi: 10.1016/j.bbrc.2019.05.113

Liu, X., Miao, J., Wang, C., Zhou, S., Chen, S., Ren, Q., et al. (2020). Tubule-derived exosomes play a central role in fibroblast activation and kidney fibrosis. Kidney Int. 97, 1181-1195. doi: 10.1016/j.kint.2019.11.026

Lou, G., Chen, Z., Zheng, M., and Liu, Y. (2017). Mesenchymal stem cell-derived exosomes as a new therapeutic strategy for liver diseases. Exp. Mo. Med. 49, e346-e346. doi: 10.1038/emm.2017.63

Lu, M., Yuan, S., Li, S., Li, L., Liu, M., and Wan, S. (2019). The exosome-derived biomarker in atherosclerosis and its clinical application. J. Cardiovasc. Transl. Res. 12, 68-74. doi: 10.1007/s12265-018-9796-y

Mauer, J., Chaurasia, B., Goldau, J., Vogt, M. C., Ruud, J., Nguyen, K. D., et al. (2014). Signaling by IL-6 promotes alternative activation of macrophages to limit endotoxemia and obesity-associated resistance to insulin. Nat. Immunol. 15, 423-430. doi: 10.1038/ni.2865

Mazur-Bialy, A. I. (2019). Superiority of the non-glycosylated form over the glycosylated form of irisin in the attenuation of adipocytic meta-inflammation: a potential factor in the fight against insulin resistance. Biomolecules 9:394. doi: 10.3390/biom9090394

McBride, J. D., Rodriguez-Menocal, L., Guzman, W., Candanedo, A., GarciaContreras, M., and Badiavas, E. V. (2017). Bone marrow mesenchymal stem cell-derived CD63(+) exosomes transport wnt3a exteriorly and enhance dermal fibroblast proliferation. Migration, and Angiogenesis In Vitro. Stem Cells Dev. 26, 1384-1398. doi: 10.1089/scd.2017.0087

McKiernan, J., Donovan, M. J., Margolis, E., Partin, A., Carter, B., Brown, G., et al. (2018). A prospective adaptive utility trial to validate performance of a novel urine exosome gene expression assay to predict high-grade prostate cancer in patients with prostate-specific Antigen $2-10 \mathrm{ng} / \mathrm{ml}$ at initial biopsy. Eur. Urol. 74, 731-738. doi: 10.1016/j.eururo.2018.08.019

McQuaid, S. E., Hodson, L., Neville, M. J., Dennis, A. L., Cheeseman, J., Humphreys, S. M., et al. (2011). Downregulation of adipose tissue fatty acid trafficking in obesity: a driver for ectopic fat deposition? Diabetes $60,47-55$. doi: $10.2337 / \mathrm{db} 10-0867$

Mleczko, J., Ortega, F. J., Falcon-Perez, J. M., Wabitsch, M., Fernandez-Real, J. M., and Mora, S. (2018). Extracellular vesicles from hypoxic adipocytes and obese subjects reduce insulin-stimulated glucose uptake. Mol. Nutr. Food Res. 62:1700917. doi: 10.1002/mnfr.201700917

Mullins, R. J., Mustapic, M., Goetzl, E. J., and Kapogiannis, D. (2017). Exosomal biomarkers of brain insulin resistance associated with regional atrophy in Alzheimer's disease. Hum. Brain Mapp. 38, 1933-1940. doi: 10.1002/hbm.23494
Mundi, M. S., Koutsari, C., and Jensen, M. D. (2014). Effects of increased free fatty acid availability on adipose tissue fatty acid storage in men. J. Clin. Endocrinol. Metab. 99, E2635-E2642. doi: 10.1210/jc.2014-2690

Muraro, P. A., Martin, R., Mancardi, G. L., Nicholas, R., Sormani, M. P., and Saccardi, R. (2017). Autologous haematopoietic stem cell transplantation for treatment of multiple sclerosis. Nat. Rev. Neurol. 13, 391-405. doi: 10.1038/ nrneurol.2017.81

Nie, Y., Sato, Y., Garner, R. T., Kargl, C., Wang, C., Kuang, S., et al. (2019). Skeletal muscle-derived exosomes regulate endothelial cell functions via reactive oxygen species-activated nuclear factor-kappaB signalling. Exp. Physiol. 104, 12621273. doi: 10.1113/EP087396

Olefsky, J. M., and Glass, C. K. (2010). Macrophages, inflammation, and insulin resistance. Annu. Rev. Physiol. 72, 219-246. doi: 10.1146/annurev-physiol021909-135846

Pan, Y., Hui, X., Hoo, R. L. C., Ye, D., Chan, C. Y. C., Feng, T., et al. (2019). Adipocyte-secreted exosomal microRNA-34a inhibits M2 macrophage polarization to promote obesity-induced adipose inflammation. J. Clin. Investig. 129, 834-849. doi: 10.1172/jci123069

Seo, W., Eun, H. S., Kim, S. Y., Yi, H. S., Lee, Y. S., Park, S. H., et al. (2016). Exosome-mediated activation of toll-like receptor 3 in stellate cells stimulates interleukin-17 production by gammadelta $\mathrm{T}$ cells in liver fibrosis. Hepatology 64, 616-631. doi: 10.1002/hep.28644

Sharma, R., Kumari, M., Prakash, P., Gupta, S., and Tiwari, S. (2020). Phosphoenolpyruvate carboxykinase in urine exosomes reflect impairment in renal gluconeogenesis in early insulin resistance and diabetes. Am. J. Physiol. Renal. Physiol. 318, F720-F731. doi: 10.1152/ajprenal.00507.2019

Shem-Tov, N., Peczynski, C., Labopin, M., Itälä-Remes, M., Blaise, D., LabussièreWallet, H., et al. (2020). Haploidentical vs. unrelated allogeneic stem cell transplantation for acute lymphoblastic leukemia in first complete remission: on behalf of the ALWP of the EBMT. Leukemia 34, 283-292. doi: 10.1038/ s41375-019-0544-3

Shim, Y. S., Kang, M. J., Oh, Y. J., Baek, J. W., Yang, S., and Hwang, I. T. (2017). Fetuin-A as an alternative marker for insulin resistance and cardiovascular risk in prepubertal children. J. Atherosc. Thromb. 24, 1031-1038. doi: 10.5551/jat. 38323

Sohn, W., Kim, J., Kang, S. H., Yang, S. R., Cho, J. Y., Cho, H. C., et al. (2015). Serum exosomal microRNAs as novel biomarkers for hepatocellular carcinoma. Exp. Mol. Med. 47:e184. doi: 10.1038/emm.2015.68

Song, M., Han, L., Chen, F. F., Wang, D., Wang, F., Zhang, L., et al. (2018). Adipocyte-derived exosomes carrying sonic hedgehog mediate M1 macrophage polarization-induced insulin resistance via Ptch and PI3K Pathways. Cell Physiol. Biochem. 48, 1416-1432. doi: 10.1159/000492252

Steculorum, S. M., Ruud, J., Karakasilioti, I., Backes, H., Engstrom Ruud, L., Timper, K., et al. (2016). AgRP neurons control systemic insulin sensitivity via myostatin expression in brown adipose tissue. Cell 165, 125-138. doi: 10.1016/ j.cell.2016.02.044

Stenvers, D. J., Scheer, F. A. J. L., Schrauwen, P., la Fleur, S. E., and Kalsbeek, A. (2018). Circadian clocks and insulin resistance. Nat. Rev. Endocrinol. 15, 75-89. doi: 10.1038/s41574-018-0122-1

Su, T., Xiao, Y., Xiao, Y., Guo, Q., Li, C., Huang, Y., et al. (2019). Bone marrow mesenchymal stem cells-derived exosomal MiR-29b-3p regulates aging-associated insulin resistance. ACS Nano 13, 2450-2462. doi: 10.1021/ acsnano.8b09375

Sun, Y., Shi, H., Yin, S., Ji, C., Zhang, X., Zhang, B., et al. (2018). Human mesenchymal stem cell derived exosomes alleviate Type 2 diabetes mellitus by reversing peripheral insulin resistance and relieving beta-cell destruction. ACS Nano 12, 7613-7628. doi: 10.1021/acsnano.7b07643

Templeman, N. M., Skovsø, S., Page, M. M., Lim, G. E., and Johnson, J. D. (2017). A causal role for hyperinsulinemia in obesity. J. Endocrinol. 232, R173-R183. doi: 10.1530/joe- 16-0449

Titchenell, P. M., Lazar, M. A., and Birnbaum, M. J. (2017). Unraveling the regulation of hepatic metabolism by insulin. Trends Endocrinol.Metab. 28, 497-505. doi: 10.1016/j.tem.2017.03.003

Tokarz, V. L., MacDonald, P. E., and Klip, A. (2018). The cell biology of systemic insulin function. J. Cell Biol. 217, 2273-2289. doi: 10.1083/jcb.201802095

Vlavcheski, F., and Tsiani, E. (2018). Attenuation of free fatty acid-induced muscle insulin resistance by rosemary extract. Nutrients 10:1623. doi: 10.3390/ nu10111623 
Wakabayashi, T., Yamaguchi, K., Matsui, K., Sano, T., Kubota, T., Hashimoto, T., et al. (2019). Differential effects of diet- and genetically-induced brain insulin resistance on amyloid pathology in a mouse model of Alzheimer's disease. Mol. Neurodegen. 14:15. doi: 10.1186/s13024-019-0315-7

Wang, L., Zhang, B., Zheng, W., Kang, M., Chen, Q., Qin, W., et al. (2017). Exosomes derived from pancreatic cancer cells induce insulin resistance in C2C12 myotube cells through the PI3K/Akt/FoxO1 pathway. Sci. Rep. 7:5384. doi: 10.1038/s41598-017-05541-4

Willis, G. R., Fernandez-Gonzalez, A., Anastas, J., Vitali, S. H., Liu, X., Ericsson, M., et al. (2018). Mesenchymal stromal cell exosomes ameliorate experimental bronchopulmonary dysplasia and restore lung function through macrophage immunomodulation. Am. J. Respir. Crit. Care Med. 197, 104-116. doi: 10.1164/ rccm.201705-0925OC

Wortzel, I., Dror, S., Kenific, C. M., and Lyden, D. (2019). Exosome-mediated metastasis: communication from a distance. Dev. Cell 49, 347-360. doi: 10.1016/ j.devcel.2019.04.011

Wu, C. X., and Liu, Z. F. (2018). Proteomic profiling of sweat exosome suggests its involvement in skin immunity. J. Invest. Dermatol. 138, 89-97. doi: 10.1016/j. jid.2017.05.040

Wu, J., Dong, T., Chen, T., Sun, J., Luo, J., He, J., et al. (2020). Hepatic exosomederived miR-130a-3p attenuates glucose intolerance via suppressing PHLPP2 gene in adipocyte. Metabolism 103:154006. doi: 10.1016/j.metabol.2019.154006

Xia, C., Zeng, Z., Fang, B., Tao, M., Gu, C., Zheng, L., et al. (2019). Mesenchymal stem cell-derived exosomes ameliorate intervertebral disc degeneration via antioxidant and anti-inflammatory effects. Free Radic. Biol. Med. 143, 1-15. doi: 10.1016/j.freeradbiomed.2019.07.026

Xie, C., Ji, N., Tang, Z., Li, J., and Chen, Q. (2019). The role of extracellular vesicles from different origin in the microenvironment of head and neck cancers. Mol. Cancer 18:83. doi: 10.1186/s12943-019-0985-3

Xu, F., Zhong, J. Y., Lin, X., Shan, S. K., Guo, B., Zheng, M. H., et al. (2020). Melatonin alleviates vascular calcification and ageing through exosomal miR204/miR-211 cluster in a paracrine manner. J. Pineal Res. 68:e12631. doi: 10. $1111 /$ jpi.12631

Xu, H., Du, X., Xu, J., Zhang, Y., Tian, Y., Liu, G., et al. (2020). Pancreatic beta cell microRNA-26a alleviates type 2 diabetes by improving peripheral insulin sensitivity and preserving beta cell function. PLoS Biol. 18:e3000603. doi: 10. 1371/journal.pbio.3000603

Xu, X., Krumm, C., So, J. S., Bare, C. J., Holman, C., Gromada, J., et al. (2018). Preemptive activation of the integrated stress response protects mice from dietinduced obesity and insulin resistance by fibroblast growth factor 21 induction. Hepatology 68, 2167-2181. doi: 10.1002/hep.30060
Xue, M., Chen, W., Xiang, A., Wang, R., Chen, H., Pan, J., et al. (2017). Hypoxic exosomes facilitate bladder tumor growth and development through transferring long non-coding RNA-UCA1. Mol. Cancer 16:143. doi: 10.1186/ s12943-017-0714-8

Ying, W., Riopel, M., Bandyopadhyay, G., Dong, Y., Birmingham, A., Seo, J. B., et al. (2017). Adipose tissue macrophage-derived exosomal miRNAs can modulate in vivo and in vitro insulin sensitivity. Cell 171, 372.e12-384.e12. doi: 10.1016/j.cell.2017.08.035

Yu, Y., Du, H., Wei, S., Feng, L., Li, J., Yao, F., et al. (2018). Adipocytederived exosomal MiR-27a induces insulin resistance in skeletal muscle through repression of PPAR $\gamma$. Theranostics 8, 2171-2188. doi: 10.7150/thno. 22565

Zeng, Z., Li, Y., Pan, Y., Lan, X., Song, F., Sun, J., et al. (2018). Cancer-derived exosomal miR-25-3p promotes pre-metastatic niche formation by inducing vascular permeability and angiogenesis. Nat. Commun. 9:5395. doi: 10.1038/ s41467-018-07810-w

Zhang, Y., Kim, M. S., Jia, B., Yan, J., Zuniga-Hertz, J. P., Han, C., et al. (2017). Hypothalamic stem cells control ageing speed partly through exosomal miRNAs. Nature 548, 52-57. doi: 10.1038/nature23282

Zhao, H., Shang, Q., Pan, Z., Bai, Y., Li, Z., Zhang, H., et al. (2018). Exosomes from adipose-derived stem cells attenuate adipose inflammation and obesity through polarizing M2 macrophages and beiging in white adipose tissue. Diabetes 67, 235-247. doi: 10.2337/db17-0356

Zhao, L., Chen, S., Shi, X., Cao, H., and Li, L. (2018). A pooled analysis of mesenchymal stem cell-based therapy for liver disease. Stem Cell Res. Ther. 9:72. doi: 10.1186/s13287-018-0816-2

Zhao, Y., and Xu, J. (2018). Synovial fluid-derived exosomal lncRNA PCGEM1 as biomarker for the different stages of osteoarthritis. Int. Orthop. 42, 2865-2872. doi: 10.1007/s00264-018-4093-6

Conflict of Interest: The authors declare that the research was conducted in the absence of any commercial or financial relationships that could be construed as a potential conflict of interest.

Copyright (c) 2021 Lei, Lin, Xu, Shan, Guo, Li, Zheng, Wang, Xu and Yuan. This is an open-access article distributed under the terms of the Creative Commons Attribution License (CC BY). The use, distribution or reproduction in other forums is permitted, provided the original author(s) and the copyright owner(s) are credited and that the original publication in this journal is cited, in accordance with accepted academic practice. No use, distribution or reproduction is permitted which does not comply with these terms. 